\title{
Manejo integrado del mal de Panamá [Fusarium oxysporum Schlechtend.: Fr. sp. cubense (E.F. SM.) W.C. Snyder \& H.N. Hansen]: una revisión
}

\section{Integrated management of Panama disease [Fusarium oxysporum Schlechtend.: Fr. f. sp. cubense (E.F. SM.) W.C. Snyder \& H.N. Hansen]: a review}

\author{
Silvia Patricia López-Zapata ${ }^{1}$; Jairo Castaño-Zapata ${ }^{2}$
}

\begin{abstract}
1Agrónoma, c.M.Sc. Fitopatología. Universidad de Caldas, Facultad de Ciencias Agropecuarias. Manizales, Colombia; e-mail: sipaloza@yahoo.es; Dhttps:// orcid.org/000-0001-6834-6794

${ }^{2}$ Ing. Agrónomo, Ph.D. Universidad de Caldas, Departamento de Producción Agropecuaria, Facultad de Ciencias Agropecuarias. Manizales, Colombia; e-mail: jairo.castano_z@ucaldas.edu.co; Dhttps://orcid.org/000-0002-3070-3343
\end{abstract}

Como citar: López-Zapata, S.P.; Castaño-Zapata, J. 2019. Manejo integrado del mal de Panamá [Fusarium oxysporum Schlechtend.: Fr. sp. cubense (E.F. SM.) W.C. Snyder \& H.N. Hansen]: una revisión. Rev. U.D.C.A Act. \& Div. Cient. 22(2):e1240. https://doi.org/10.31910/ rudca.v22.n2.2019.1240

Artículo de acceso abierto publicado por Revista U.D.C.A Actualidad \& Divulgación Científica, bajo una licencia Creative Commons CC BY-NC 4.0

Recibido: Septiembre 30 de 2018

Aceptado: Junio 17 de 2019

\section{RESUMEN}

El banano es un frutal muy importante, a nivel mundial y en Colombia es el tercer producto de exportación. Una de las enfermedades limitantes del cultivo es el mal de Panamá, ocasionado por Fusarium oxysporum f. sp. cubense. La enfermedad es considerada devastadora, por las pérdidas que ocasionó en Gros Michel, obligando al reemplazo de este cultivar por Cavendish, el cual, está siendo amenazado por la raza tropical 4 del hongo que, aunque aún no se encuentra en el país, representa una amenaza mundial. El manejo de la enfermedad, se ha basado en la exclusión del patógeno, evitando el ingreso a áreas sanas y usando variedades resistentes, pero la naturaleza del patógeno ha demostrado que estas medidas no son suficientes y que es necesario considerar otras estrategias. El Manejo Integrado de Enfermedades MIE, en su filosofía, busca conjugar opciones en pro del cultivo para garantizar, además de la sanidad, altos rendimientos e inocuidad. Esta revisión compila información de los resultados de investigación, obtenidos mediante el uso de diferentes medidas y que sean factibles de incluirse dentro de un programa de manejo integrado del mal de Panamá, enmarcándolas dentro del modelo logístico propuesto por Van der Plank y enfatizando en la necesidad de realizar más investigación, para obtener materiales resistentes y llevar a cabo evaluaciones en el campo, que demuestren, de manera más efectiva, la viabilidad de las prácticas propuestas.

Palabras clave: hongo; raza 4; banano; musáceas: epidemiología.

\section{ABSTRACT}

Banana is a very important fruit worldwide and in Colombia it is the third export product. One of the diseases limiting the crop is the Panama disease caused by Fusarium oxysporum f. sp. cubense. The disease is considered devastating because of the losses it caused in Gros Michel, forcing the replacement of this cultivar by Cavendish, which is being threatened by the tropical race 4 of the fungus, which although not yet found in the country, represents a global threat. The management of the disease has been based on the 
exclusion of the pathogen, avoiding entry into healthy areas and using resistant varieties, but the nature of the pathogen has shown that these measures are not enough and that other strategies need to be considered. The Integrated Management of Diseases, MIE, in its philosophy seeks to combine options in favor of the crop to guarantee besides the health, high yields and innocuousness. This review compiles information on the research results obtained through the use of different measures and feasible to be included in an integrated management program of the Panama disease, framing them within the logistic model proposed by Van der Plank and emphasizing the need to conduct more research to obtain resistant materials and carry out evaluations in the field that more effectively demonstrate the feasibility of the proposed practices.

Keywords: fungus; race 4; banana; Musaceae; epidemiology.

\section{INTRODUCCIÓN}

El banano es considerado el cuarto alimento más consumido en el mundo después del arroz, el trigo y el maíz (Akila et al. 2011; Siddhesh et al. 2015). Muchos de los cultivares son híbridos naturales, seleccionados por los primeros agricultores y, actualmente, los bananos comestibles provienen de dos especies diploides Musa acuminata (AA) y M. balbisiana (BB) (Perrier et al. 2011). Se conocen más de 50 subgrupos, pero solo Gros Michel y Cavendish, producen la mayor cantidad de fruta, siendo Cavendish el más importante (Ploetz, 2015b). En América Latina y el Caribe, se concentra cerca del 28\% de la producción mundial ( $M$. acuminata AA), con tres de los diez productores y exportadores más importantes en el mundo (Dita et al. 2013).

Dentro de las enfermedades limitantes del cultivo está el mal de Panamá, causado por Fusarium oxysporum Schlechtend. f. sp. cubense (E.F. Smith) W. C. Snyder \& H. N. Hansen, siendo una de las más destructivas en banano (Akila et al. 2011; Belgrove et al. 2011; Ploetz, 2015b), por la epidemia que causó la raza 1 en América Central, a mitad del siglo XX, en "Gros Michel" (Nel et al. 2007), obligando al cambio por cultivares resistentes del tipo Cavendish (Buddenhagen, 2009). Los bananos pertenecientes a Cavendish tienen nuevamente amenazada la producción y la sostenibilidad, debido a su susceptibilidad, a una nueva variante del hongo, denominada raza tropical 4 (RT4) (Meldrum et al. 2013a; Ploetz, 2015a), hecho que debió preverse, considerando lo sucedido con Gros Michel y el riesgo de tener extensos monocultivos con clones estrechamente relacionados con la genética.

F. oxysporum hace parte de la comunidad de hongos del suelo y es considerado como componente natural de la rizosfera de las plantas; todas las cepas de la especie son saprófitas y pueden sobrevivir sobre materia orgánica; sin embargo, algunas son fitopatógenas y penetran las raíces del hospedante, induciendo marchitamientos vasculares (Fravel et al. 2003); posee, además, gran especificidad patógenohospedante (Ploetz, 2006), que ha llevado a la identificación de alrededor de 150 formas especiales y razas fisiológicas (Gordon, 2017), como F. oxysporum f. sp. cubense (Ploetz, 2015b), F. oxysporum f. sp. lycopersici (Marín-Serna et al. 2014) y F. solani f. sp. passiflorae (Ángel-García et al. 2018).

F. oxysporum f. sp. cubense (Foc), originario del Sudeste de Asia, está presente en todas las zonas productoras ( Nel et al. 2007). Es un microorganismo genética y patogénicamente diverso, del cual, se han identificado cuatro razas: la raza 1, afecta a Gros Michel y Manzano (AAB); la raza 2, ataca a los cultivares ABB, conocidos como Guineo, Cuadrado, Topocho y Chatos; la raza 3, a heliconias (Heliconia L.) y la raza 4, a cultivares susceptibles a las razas 1, 2 y del tipo Cavendish (Hennessy et al. 2005; Ploetz, 2015b). Dentro de la raza 4, se ha identificado la raza tropical (RT4) y la subtropical (ST4), ambas pertenecientes a diferentes grupos de compatibilidad vegetativa; la primera, causa enfermedad en trópicos y subtrópicos y, la segunda, solo en el subtrópico (Belgrove et al. 2011). Esta nueva variante del patógeno tiene la capacidad de infectar no solo a los bananos tipo Cavendish sino también al plátano (Shirani \& Sijun, 2018).

Aunque no existen reportes de la recombinación sexual para Foc, este es un patógeno altamente variable, que utiliza otros mecanismos, como mutación, elementos transponibles en el genoma, anastomosis hifal, flujo de genes y selección (Costa et al. 2014), que dificultan su manejo y ponen de manifiesto la gravedad de sus ataques que sobrepasan, en tiempo y en espacio, la capacidad humana de desarrollar variedades resistentes o tolerantes.

Externamente, Foc produce dos tipos de síntomas: síndrome de la hoja verde y síndrome de la hoja amarilla. En el primero, las hojas permanecen verdes hasta que el peciolo se dobla y colapsa; en el segundo, el más común, los bordes de las hojas adultas se tornan amarillas y se dividen longitudinalmente, avanzando, de manera gradual, hacia las hojas jóvenes (Pérez et al. 2014) (Figura 1a). En genotipos susceptibles, las esporas del hongo germinan estimuladas por compuestos orgánicos, que son liberados por las raíces del hospedante; la penetración la realiza directamente por la cofia de la raíz, con la ayuda de enzimas degradativas o mediante el crecimiento intercelular, momento a partir del cual procede la colonización, desde el ápice de la raíz (Gordon, 2017). U una vez el hongo avanza por el tejido vascular, causa una decoloración que se extiende hacia el pseudotallo (Ploetz, 2006; Pérez et al. 2014) (Figura 1b). Los síntomas son más evidentes entre 5 y 6 meses después de la siembra, caracterizados por la muerte de las plantas y pérdidas en el rendimiento (Ploetz, 2006; Akila et al. 2011; Sun et al. 2011).

El patógeno, en medio de cultivo, produce micelio de color blanco con fondo rojizo (Figura 1c), que se acentúa con el tiempo (Figura 1d), produciendo microconidios, con forma elíptica, oval o de riñón, usualmente, sin septos (Figura 2a); macroconidios, con forma de hoz y tres septos (Figura 2b) y clamidosporas (Figura 2c), con paredes lisas o ásperas, formadas individualmente, en pares o en cadenas (Leslie \& Summerell, 2006; Pérez et al. 2014; Siddhesh et al. 2015). Las clamidosporas pueden sobrevivir por más de 30 años en ausencia de hospedante principal (Ploetz, 2006), pero el hongo, igualmente, tiene la capacidad de infectar arvenses (Ploetz, 2015b), musáceas silvestres (Musa spp.) y heliconias (Heliconia spp.) (Pérez et al. 2014). 


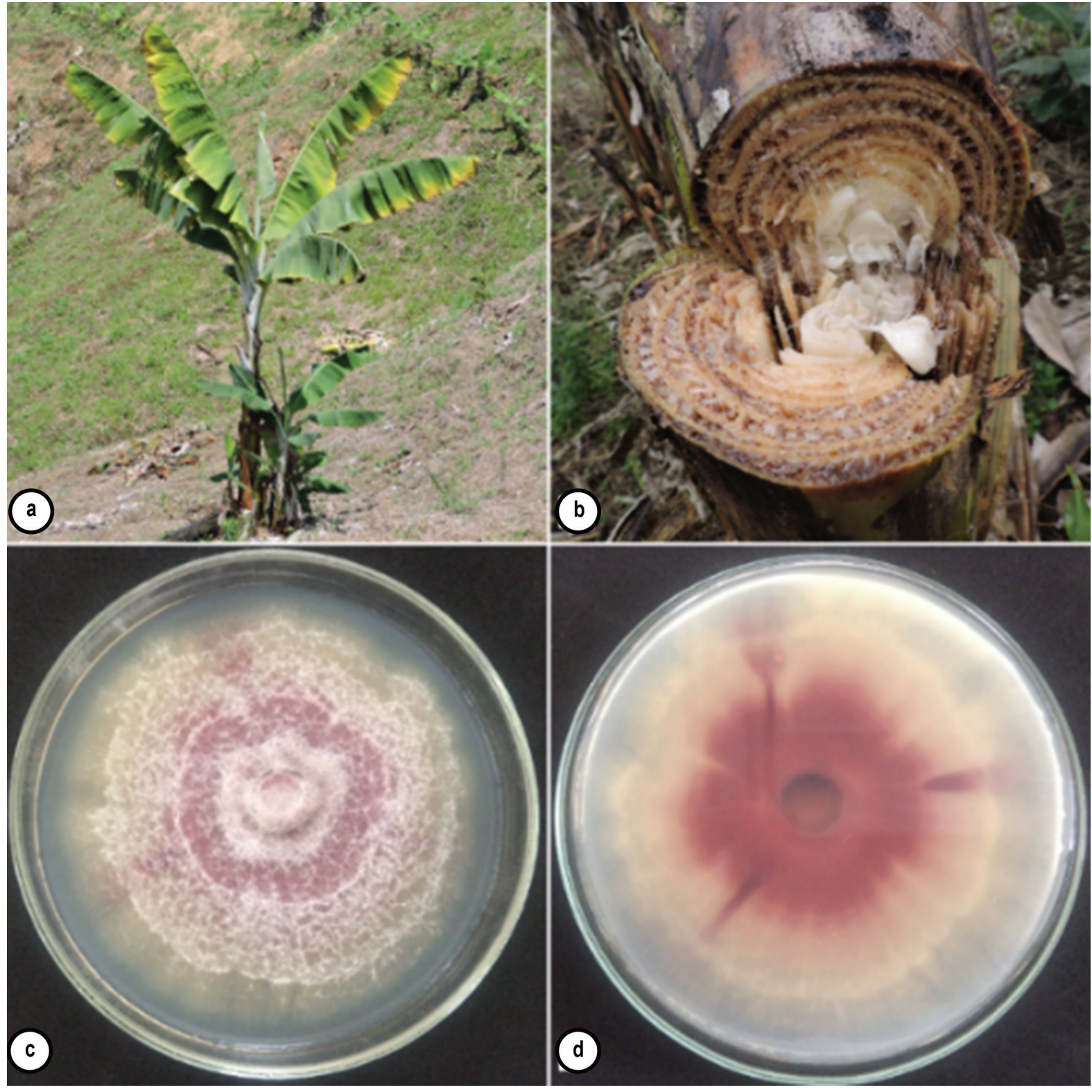

Figura 1. a. Síntomas externos del mal de Panamá; b. Necrosamiento de los haces vasculares; c-d. Crecimiento característico de Fusarium oxysporum f. sp. cubense en medio PDA. 
En Colombia, Fusarium oxysporum f. sp. cubense se detectó en rizomas e inflorescencias de Heliconias (Alarcón, 2007) y Fusarium spp., en pseudotallos, pedicelos, inflorescencias y rizomas de las variedades Salmón, Orange, Kawachi y Edge of night (López \& CastañoZapata, 2012).
La dispersión de los patógenos habitantes del suelo, como Foc, es usualmente a cortas distancias, excepto por el movimiento de plantas contaminadas que, además, permiten la introducción de nuevos alelos entre las poblaciones existentes, ocasionando grandes cambios en su estructura genética (Costa et al. 2014). En este caso, se ha documentado que la diseminación del hongo en las plantaciones se hace a través de suelo, agua, herramientas, equipos

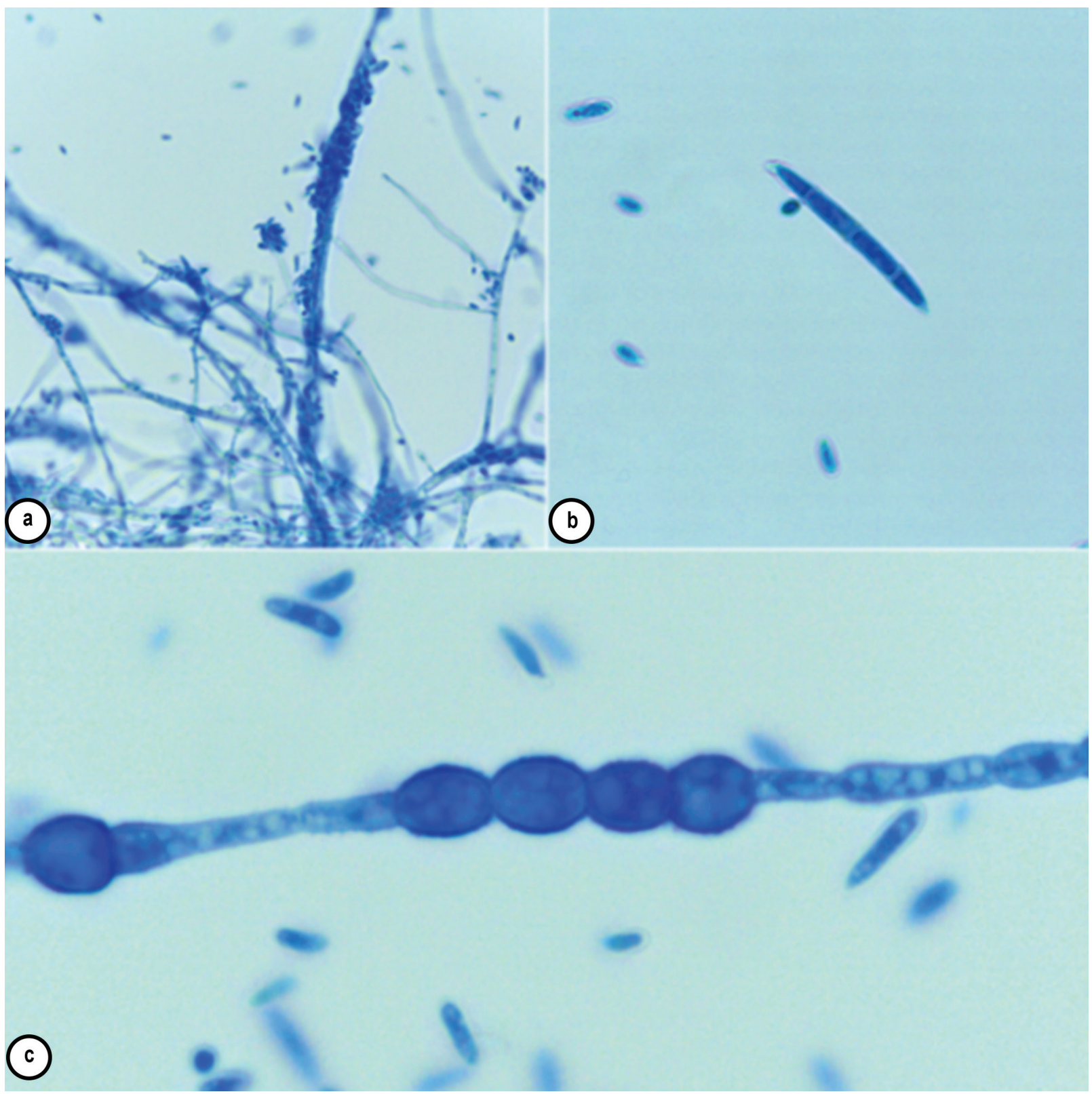

Figura 2. Estructuras reproductivas del hongo. a. Microconidios; b. Macroconidio; c. Clamidosporas en cadena. 
agrícolas, ropa, botas, material de siembra (Ploetz, 2015a), por el picudo negro (Cosmopolites sordidus Germar) (Meldrum et al. 2013a) y por nematodos fitoparásitos, como Radopholus similis (Cobb) Thorne (Pérez et al. 2014). Otras prácticas que pueden contribuir con la adaptación y la dispersión de este hongo son los agroecosistemas modernos, donde la producción bananera se caracteriza por el uso intensivo de prácticas de manejo, como riego, variedades resistentes y prácticas culturales (Costa et al. 2014).

El mal de Panamá es una enfermedad monocíclica, típica de marchitez vascular, en la que, por efecto del hongo y las respuestas del hospedante, se produce la oclusión de los vasos del xilema, impidiendo el transporte de agua y de nutrientes (Akila et al. 2011; Pérez et al. 2014). Algunas de las prácticas para el manejo de la enfermedad son preventivas, como la aplicación de fumigantes al suelo (Fravel et al. 2003), siembras en campos libres del patógeno y el empleo de semilla sana (Ploetz, 2015a).

Debido a la dificultad de controlar el hongo por su sistemicidad y como respuesta a las preocupaciones que surgen del uso inadecuado y excesivo de los productos químicos, es necesario establecer una alerta ante la inminente llegada al país de la RT4, enfatizando en la prevención, mediante la implementación de alternativas de manejo integrado (MIE), cuyos principios básicos son: exclusión o erradicación del patógeno, escape de la infección, desarrollo y uso de la resistencia genética, protección de las plantas y reducción de la incidencia de la enfermedad (Castaño-Zapata, 1994; Jiménez \& Jiménez, 2011), conceptos que pueden ser aplicados, mediante la utilización conjunta de las estrategias disponibles, reduciendo los efectos adversos del patógeno sobre un cultivo, sin disminuir la calidad y el rendimiento.

Para el mal de Panamá, se ha estimado la exclusión, como la estrategia más efectiva (Pérez, 2015), pero considerando el desconocimiento de aspectos biológicos del patógeno y ecológicos de la enfermedad, en lo que respecta a la interacción Cavendish - F. oxysporum f. sp. cubense RT4, la mejor opción es el uso de variedades resistentes (Ploetz, 2015a) que, combinadas con otras prácticas, conllevan a la aplicación del concepto de MIE, estrechamente ligado al modelo logístico de James Edward Van der Plank (1963). Éste, cuantifica la tasa de desarrollo de una enfermedad (r) y caracteriza su progreso, mediante la obtención de valores de enfermedad $(\mathbf{X})$ e inóculo inicial $\left(\mathbf{X}_{\mathbf{o}}\right)$, en un espacio de tiempo $(\mathbf{t})$, variables que se expresan mediante la ecuación $\mathbf{X}=\mathbf{X}_{\circ} \mathrm{e}^{\text {r.t. }}$, siendo $\mathrm{e}=$ la constante universal equivalente a 2,7182 .

\section{MATERIALES Y MÉTODOS}

Se realizó una búsqueda de información, a través Internet, enfatizando en las bases de datos científicas, como Dialnet, Scielo, Science direct, Scopus, Google académico y Jstor, donde se obtuvo la información relevante del tema, publicada durante 30 años, por medio de más de 80 artículos científicos, tanto en español como en inglés, con las palabras clave Fusarium oxysporum f. sp. cubense raza tropical 4, Fusarium wilt, Fusariose, Plantains, Bioactive compounds, banana production.

\section{RESULTADOS Y DISCUSIÓN}

El control de patógenos habitantes del suelo es complejo, más aún, cuando producen estructuras de resistencia, como sucede con Fusarium y aunque se han desarrollado diferentes prácticas para su manejo, incluido el control químico con fungicidas sistémicos, la naturaleza de estos productos es una amenaza, por el aumento de la resistencia del patógeno. Se ha indicado, que el manejo para todas las razas, incluida la RT4, sea similar a las empleadas para Ralstonia solanacearum (Smith), las cuales, están enfocadas, principalmente, a disminuir el inóculo inicial $\left(\mathbf{X}_{\mathbf{0}}\right)$, y consideran aspectos, como la delimitación de áreas de acceso, aplicación de fumigantes, eliminación de plantas enfermas y rotación con especies no hospedantes (Pérez et al. 2014).

Control legal. La cuarentena, cuyo efecto epidemiológico es sobre $\mathbf{X}_{\mathbf{0}}$, es una medida todavía viable en Colombia y complementa el "Plan Nacional de Foc RT4", para mantener el patógeno en la categoría de plaga cuarentenaria ausente. Este mecanismo constituye una primera e importante barrera, porque, una vez el patógeno se ha introducido en un cultivo, su erradicación es imposible (Ploetz, 2015b), ya que los esfuerzos para contener la enfermedad en los países donde está presente, a través de la cuarentena inter o intra nacional, no han sido efectivos, como ha sido evidente por la diseminación del hongo entre continentes, países y regiones (Dale et al. 2017).

En todo caso, las medidas a establecer deben ser coordinadas y en cooperación con los entes directamente involucrados, como son productores, importadores y exportadores; la vigilancia constante es fundamental para la detección temprana de focos de la enfermedad, mediante el flujo de información continuo, en cooperación con las instituciones gubernamentales involucradas, con el asesoramiento de especialistas en micología, para la identificación correcta del patógeno y la raza presente, sumado a la investigación requerida, para mejorar o desarrollar técnicas más eficientes para la detección oportuna del hongo.

De igual manera, el material de propagación, sitios donde se produce, el tránsito de material infectado y las personas provenientes de países donde el patógeno está presente, deben ser estrictamente controlados, para facilitar el establecimiento de estrategias de manejo de la enfermedad y la implementación de medidas cuarentenarias, ante una potencial llegada de la RT4 al país.

Resistencia. La naturaleza de la enfermedad reduce las formas de manejo; usualmente, la siembra de materiales susceptibles debe provenir de semilla sana y sembrarse en suelos libres del patógeno (Ploetz, 2015a), prácticas que afectan el inóculo inicial $\left(\mathbf{X}_{\mathbf{0}}\right)$. Aun así, el uso de materiales resistentes es la estrategia más efectiva para el manejo de la enfermedad (Fravel et al. 2003; Costa et al. 2014; Ploetz, 2015a); estos materiales, dependiendo de su naturaleza y los genes involucrados, tienen un efecto epidemiológico sobre el inóculo inicial $\left(\mathbf{X}_{0}\right)$, si la resistencia es monogénica o sobre la tasa de desarrollo de la enfermedad (r), si es poligénica (Castaño-Zapata, 2002). 
Infortunadamente, los programas de mejoramiento genético no han logrado producir materiales con iguales o mejores características de productividad y de calidad que satisfagan las exigencias del mercado, en aspectos como la calidad de la fruta (Molina et al. 2011; Wang et al. 2013), pero es posible que estos materiales tengan aceptación en lugares donde las condiciones de pos-cosecha y la tolerancia a diferentes sabores no sean relevantes (Ploetz, 2015a) o donde el cambio no represente un riesgo para la economía nacional y regional, como ocurre en la región de Urabá - Antioquia, Colombia, en donde el impacto económico y social sería de grandes proporciones.

La Fundación Hondureña de Investigación Agrícola, FHIA, desarrolló materiales de banano resistentes a $F$. oxysporum f. sp. cubense RT4 (Aguilar, 2013); incluyeron, también, híbridos resistentes a la raza 1 y subtropical 4 y otros con mayor resistencia que el cultivar Gros Michel (Smith et al. 2014). De igual manera, en Asia se han logrado nuevas líneas altamente resistentes a Foc RT4, con rendimientos y calidad comparables a las variedades comerciales (Molina et al. 2011; Molina et al. 2016; Dale et al. 2017), en donde varios genotipos de parientes silvestres de banano han mostrado diferentes niveles de resistencia a la raza 4 del hongo (Li et al. 2014; Zuo et al. 2018).

En subespecies de $M$. acuminata, como burmannica, microcarpa y siamea, se han encontrado alelos con resistencia a Foc R1; en la subsp. Malaccensis, a las razas 1 y 4 (Shirani \& Sijun, 2018) y en M. basjoo y M. itinerans, alta resistencia a Foc TR4; aunque por la riqueza genética que se encuentra en los materiales silvestres y la cual puede ser objeto de variación en el grado de resistencia, se requieren selecciones cuidadosas (Li et al. 2014).

Control cultural. Para evitar la dispersión de la raza 4, se ha recomendado la eliminación de un anillo simple o doble alrededor de las plantas enfermas (Meldrum et al. 2013a). Esta práctica, comúnmente usada para el manejo de la enfermedad, tiene efecto epidemiológico en la reducción del inóculo inicial $\left(\mathbf{X}_{\mathbf{0}}\right)$, además de evitar la diseminación del patógeno hacia áreas libres, pero necesita complementarse con otras estrategias para ampliar su efectividad, debido a que, conociendo la naturaleza del patógeno y la enfermedad, no garantiza por sí sola un método de control efectivo.

La rotación de cultivos, que igualmente reduce el inóculo inicial $\left(\mathbf{X}_{0}\right)$, tiene limitaciones por la capacidad del hongo para sobrevivir por largo tiempo, mediante clamidosporas, aún en ausencia del hospedante principal; no obstante, otras estrategias, como la siembra intercalada o rotación con especies no hospedantes del hongo, como yuca (Manihot esculenta Crantz) (Buddenhagen, 2009), puerro chino (Allium tuberosum Rottler ex Spreng.) (Huang et al. 2012; Zhang et al. 2013b) y piña [Ananas comosus (L.) Merr.] (Wang et al. 2015), reducen la incidencia de la enfermedad; del mismo modo, el uso de cubierta vegetal al suelo y alrededor de la base de las plantas, disminuye la incidencia de Foc ( $\mathbf{X}_{\mathbf{o}}$ ), alargando el periodo de desarrollo de los síntomas de la enfermedad (r) (Pattison et al. 2014).

Otras prácticas que tienen efecto sobre el inóculo inicial $\left(\mathbf{X}_{\mathbf{0}}\right)$ son el uso de desinfestantes de superficie sobre botas, herramientas y maquinaria (Nel et al. 2007). Está confirmada la efectividad del cloruro de polimetilamonio (Sporekill $($ ), sobre Foc, después de 30 segundos de exposición (Meldrum et al. 2013b); la esterilización del suelo con calor, colocando cascarilla de arroz en los sitios donde se han observado plantas enfermas (Ploetz, 2015a) o el aislamiento de sitios afectados con láminas de aluminio o zinc, para luego quemarlos, incluyendo los rizomas de las plantas afectadas (Pérez et al. 2014).

La solarización del suelo es un tratamiento efectivo y representa una alternativa ante la ausencia de estrategias seguras para el control de patógenos; su efecto está en la reducción del inóculo inicial $\left(\mathrm{X}_{0}\right)$ y se puede mejorar en combinación con un fumigante al suelo, aunque estos últimos son poco efectivos, debido al ciclo del cultivo (Herbert \& Marx, 1990); sin embargo, Hermanto et al. (2012) demostraron que en un suelo infectado con Foc RT4, sometido únicamente al tratamiento físico, la aparición de la enfermedad se retrasó más tiempo que rotando con maíz durante dos siembras consecutivas y barbecho durante 10 meses.

El manejo de arvenses, se debe considerar dentro de un programa de MIE, ya que actúan como hospedantes alternos del hongo, afectando el inóculo inicial $\left(\mathbf{X}_{\mathbf{0}}\right)$ y la tasa de desarrollo $(\mathbf{r})$, al ser portadoras endofíticas del hongo y contribuyendo a la acumulación de inóculo, especialmente gramíneas (Pérez, 2015; Deltour et al. 2017). Especies de la familia Asteraceae, como el rabo de buey [Cyanthilium cinereum (L.) H.Ros], de la cual, se ha aislado la raza 4, merece atención (Hennessy et al. 2005).

Otro efecto del control cultural es la reducción de la tasa de desarrollo de la enfermedad (r). Al respecto, se reporta que es posible disminuir la germinación de los conidios, con la aplicación de fuentes de calcio ( $\mathrm{Ca}$ ) y de hierro (Fe), la modificación de la temperatura del suelo, el contenido de agua y pH (Peng et al. 1999) y se puede evitar la penetración del hongo, a través de las raíces, mediante fertilización a base de amonio $\left(\mathrm{NH}_{4}\right)$, como se ha demostrado in vitro y en cultivos hidropónicos (Zhang et al. 2013a).

Control biológico. Debido al uso inadecuado de fungicidas y la capacidad del patógeno de adquirir resistencia a estos, el control biológico toma vigencia en los programas de MIE (Fravel et al. 2003) y su efecto se refleja sobre el inóculo inicial $\left(\mathbf{X}_{\mathbf{0}}\right)$ y la tasa de desarrollo de la enfermedad (r). La literatura científica indica a los suelos portadores de aislamientos no patogénicos de Fusarium spp. o pertenecientes a otra forma especial, actuando como supresivos a cepas patogénicas (Amir \& Alabouvette, 1993), estableciendo competencia por carbono (Fravel et al. 2003), nutrientes, sitios de infección o inducción de resistencia sistémica (Forsyth et al. 2006); efectos similares a los inducidos por bacterias promotoras del crecimiento vegetal (Lian et al. 2008; Fishal et al. 2010) y cepas no patogénicas de Bacillus y Pseudomonas (Saravanan et al. 2003; Lian et al. 2008; Thangavelu \& Jayanthi, 2009; Fishal et al. 2010). De igual manera, los compuestos volátiles de hongos endófitos aislados de Musa spp. han mostrado un efecto inhibitorio altamente significativo sobre Foc R4 (Ting et al. 2010). 
Respecto a la resistencia inducida y aunque se conocen efectos de activación enzimática por el metil jasmonato sobre plántulas de banano inoculadas con Foc RT4 (Sun et al. 2011), aún se considera que no están bien dilucidados los mecanismos, por los cuales, el jasmonato contribuye en la reducción de la enfermedad (Shirani \& Sijun, 2018). En el mismo sentido, el ácido salicílico ha demostrado tener un papel importante en inducir resistencia en plantas de banano a Foc RT4 (Wang et al. 2014) y en plantas de Cavendish inoculadas con Foc R1; se ha observado, la acumulación endógena de esta última hormona vegetal y la activación de la expresión génica de la misma, mejorando la resistencia a Foc RT4 (Wu et al. 2013).

Referente a otros microorganismos, se ha documentado que B. subtilis inhibe el crecimiento micelial, coloniza los tejidos de la planta, disminuye la decoloración vascular y la incidencia de la enfermedad (Sun et al. 2011); asimismo, Wang et al. (2013) comprobaron que la combinación de B. amyloliquefaciens W19 y compuestos orgánicos, además de disminuir la incidencia de la marchitez, proporcionan nutrientes a las plantas de banano, resultados que se interpretan, desde el punto de vista epidemiológico, como una reducción en la tasa de desarrollo (r) del mal de Panamá, efecto que también ha sido demostrado con las bacterias biocontroladoras y biofertilizantes Burkholderia sp. y Herbaspirillum sp. (Weber et al. 2007); Gamma-proteobacterias (Köberl et al. 2017); con el hongo micorrízico arbuscular Gigaspora margarita (Borges et al. 2007); con Trichoderma harzianum y Glomus mosseae (Mohandas et al. 2010).

Estudios que muestran efecto sobre el inoculo inicial $\left(\mathrm{X}_{0}\right)$ y que involucren el uso de organismos vivos, reportan a Serratia marcescens como inductora de producción de enzimas involucradas en la defensa de las plantas de banano, suprimiendo la incidencia de la enfermedad (Ting et al. 2010) y Streptomyces griseus afectando el desarrollo de Foc R4, al inducir la generación de hifas distorsionadas y protuberantes, inhibiendo la formación de esporas y degradando la pared celular del hongo (Zacky \& Ting, 2013).

Getha \& Vikineswary (2002) demostraron que Streptomyces violaceusniger $\mathrm{G} 10$ inhibe el crecimiento micelial, distorsiona, induce ramificación excesiva, causa lisis en los extremos de las hifas e impide la germinación de los conidios; efectos similares a los observados por Thangavelu \& Gopi (2015), con las bacterias endofíticas Pseudomonas putida, Achromobacter sp., Rhizobium sp., Ochromobactrum sp. y Bacillus flexus 'Tvpr1, que inhiben la germinación de los conidios y el crecimiento micelial del hongo; igualmente, se ha demostrado que el quitosano inhibe el crecimiento micelial, esporulación y germinación del hongo (Al-hetar et al. 2011).

Es obvio el efecto de los microorganismos benéficos sobre las cepas patogénicas, pero también es claro que cada uno de los resultados deben ser validados bajo las condiciones propias del cultivo, donde se identifiquen los organismos endófitos o rizosféricos y que, potencialmente, causen los mismos efectos reportados; además, existe el desafío de producirlos masivamente, considerando que son organismos vivos, que pueden cambiar su biología bajo condiciones adversas, de modo que, para garantizar su efectividad y su patogenicidad, se requiere de muchos años de investigación.
Control químico. Los fungicidas químicos afectan directamente la tasa de desarrollo de la enfermedad (r) y han sido la estrategia más ampliamente usada para el control de enfermedades, por la facilidad en su uso, la consecución y por la rapidez con que se observan los resultados, pero no es nueva la preocupación que causa el uso inadecuado e indiscriminado que se les ha dado, sobre aspectos, como la salud humana, la vida silvestre y el ambiente, esto agregado a las mutaciones y la resistencia que ha adquirido el hongo a los fungicidas (Ramu et al. 2016) y sus efectos genotóxicos, sobre algunas especies de plantas (Dane \& Dalgic, 2005).

Aunque Lakshmanan et al. (1987) demostraron que el fungicida carbendazim al 2\%, inyectado en los rizomas de banano es eficiente, el resultado puede no ser el mismo en otros países, lo que pone de manifiesto la imposibilidad, en muchos casos, de la aplicabilidad explicita de los resultados, que están en ocasiones condicionados a particularidades del clima, de la cepa patogénica o a las variedades cultivadas. Otros estudios realizados por Nel et al. (2007), concluyeron que procloraz, propiconazole, benomil y los fungicidas inhibidores de la demetilación, además de reducir la incidencia de la enfermedad y el crecimiento del hongo, aumentan los rendimientos del cultivo.

Fungicidas botánicos. Extractos de plantas medicinales, como pimienta común (Piper betle L.) (Gnanasekaran et al. 2015) y neem (Aqadirachta indica A. Juss.), tienen actividad antifúngica sobre F. oxysporum f. sp. cubense (Mengane \& Kamble, 2014); asimismo, los aceites esenciales de clavos [Eugenia aromatica (L.) Baill. y Sysygium aromaticum (L.) Merr. \& L.M.Perry (L.)], de canela (Cinnamomum zeylanicum Blume.), de hojas de Zimmu (Allium cepa L. x Allium sativum L.) y de puerro chino [Allium ampeloprasum var. porrum (L.) Gay] tienen efecto inhibitorio sobre el crecimiento micelial y germinación de conidios, incluyendo a la raza 4 (Monteiro et al. 2013; Gopi \& Thangavelu, 2014; Zuo et al. 2015; Villa et al. 2015; Juniawan et al. 2016).

Transformación genética. Este mecanismo parece ser el más eficiente y promisorio para mejorar la resistencia o tolerancia a enfermedades de plantas (Shirani \& Sijun, 2018); para ello, se han empleado diferentes técnicas, como el bombardeo de partículas o transformación de genes intervenidos por Agrobacterium tumefasciens (Ghag et al. 2014; Subramanyam et al. 2011), el uso de parámetros bioquímicos (Subramaniam et al. 2006) y la proteómica, con la cual, se han logrado identificar la expresión de proteínas reguladoras, luego de inoculaciones con Foc RT4 (Li et al. 2013).

Estas investigaciones aún siguen siendo exploratorias y no se han probado bajo condiciones de campo. En este último caso, existen algunos reportes con resultados promisorios (Pei et al. 2005), como los obtenidos por Dale et al. (2017), quienes probaron en campo qué materiales transgénicos de Cavendish mostraron resistencia a Foc RT4, conservándose libres de la enfermedad por un periodo de tres años; sin embargo, no se sabe de materiales de este tipo comercialmente disponibles (Shirani \& Sijun, 2018). 


\section{Prácticas de manejo integrado}

No son muy comunes los estudios donde se combinen diferentes estrategias de manejo del mal de Panamá; no obstante, se destacan resultados importantes, como el de Akila et al. (2011), con la mezcla de extractos metanólicos de hojas de trompeta del diablo (Datura metel L.), con cepas de Pseudomonas fluorescens y Bacillus subtilis, cuya combinación tiene un efecto sinérgico sobre la reducción de la incidencia de la enfermedad; lo mismo que la incorporación al suelo de residuos de maíz o de arroz en combinación con la inundación o saturación de agua, que inactivan a F. oxysporum $\mathrm{f}$. sp. cubense (Wen et al. 2015). Asimismo, la aplicación conjunta de fertilizantes, a base de compost maduro y Bacillus amyloliquefaciens $\mathrm{NJN}-6$, con efecto supresivo del mal de Panamá (Shen et al. 2013), efectos similares a los obtenidos con la mezcla de estiércol de cerdo y Bacillus subtilis N11, (Zhang et al. 2014a) o Bacillus amyloliquefaciens W19 (Wang et al. 2016).

Otra alternativa es la suplementación de la fertilización con silicio (Si), solo o con cáscaras de macadamia y cepas no patogénicas del hongo, para disminuir la incidencia del mal de Panamá (Kidane \& Laing, 2010; Fortunato et al. 2012), resultado que también se ha obtenido con la integración de un agente de biocontrol, como las bacterias promotoras de crecimiento vegetal y una enmienda orgánica, como lo demostraron Zhang et al. (2014b), al mezclar compost con Paenibacillus polymyxa SQR-21, Trichoderma harzianum T37, Bacillus amyloliquefaciens N6 y Bacillus subtilis N11.

\section{CONCLUSIONES Y RECOMENDACIONES}

La identificación de métodos y de microorganismos que provean diferentes niveles de control de F. oxysporum $\mathrm{f}$. sp. cubense, se requieren para afrontar los ataques del hongo. La efectividad de las prácticas mencionadas en esta revisión (Tabla 1), representa un desafío para productores, fitopatólogos y fitomejoradores; por consiguiente, la tendencia de los estudios in vitro e in situ, aunque de gran relevancia, se deben validar bajo condiciones de campo, a través del tiempo y espacio, concordantes con las características de los materiales comerciales; considerando la larga supervivencia del hongo en el suelo, la susceptibilidad de los cultivares de banano y la ausencia de la integración de medidas efectivas para su control, quedando claro que el desarrollo de genotipos con resistencia genética, preferiblemente poligénica, es la prioridad inmediata para afrontar a este patógeno.

Tabla 1. Efecto epidemiológico de las principales prácticas de manejo del mal de Panamá, causado F. oxysporum f. sp. cubense.

\begin{tabular}{|l|c|}
\hline \multicolumn{1}{|c|}{ PRÁCTICA } & EFECTO EPIDEMIOLÓGICO \\
\hline Cuarentena & $\mathrm{X}_{\circ}$ \\
\hline Destrucción de plantas enfermas & $\mathrm{X}_{\circ}$ \\
\hline Rotación de cultivos & $\mathrm{X}_{\circ}$ \\
\hline Siembras intercaladas & $\mathrm{X}_{\circ}$ \\
\hline Desinfestación de herramientas, botas y maquinaria & $\mathrm{X}_{\circ}$ \\
\hline Esterilización del suelo con calor & $\mathrm{X}_{\circ}$ \\
\hline Solarización & $\mathrm{X}_{\circ}$ \\
\hline Fumigación del suelo & $\mathrm{X}_{\circ}$ \\
\hline Aplicación de fungicidas químicos y biológicos & $\mathrm{X}_{\mathrm{o}-\mathrm{r}}$ \\
\hline Aplicación de bacterias promotoras de crecimiento + enmiendas orgánicas & $\mathrm{r}$ \\
\hline Adición de microelementos al suelo & $\mathrm{r}$ \\
\hline Regulación del contenido de agua en el suelo & $\mathrm{r}$ \\
\hline Modificación del pH del suelo & $\mathrm{r}$ \\
\hline Fertilización nitrogenada & $\mathrm{r}$ \\
\hline Aplicación de cepas no patogénicas + residuos orgánicos & $\mathrm{r}$ \\
\hline Resistencia genética & $\mathrm{X}_{\mathrm{o}-\mathrm{r}}$ \\
\hline Manejo de arvenses & $\mathrm{X}_{\mathrm{o}-\mathrm{r}}$ \\
\hline Aplicación de microorganismos (hongos, bacterias) & $\mathrm{X}_{\mathrm{o}-\mathrm{r}}$ \\
\hline
\end{tabular}

$\mathrm{X}_{\mathrm{o}}=$ Inóculo inicial

$\mathrm{X}_{\mathrm{o}-\mathrm{r}}=$ Inóculo inicial y tasa de desarrollo de la enfermedad 
Considerando que las poblaciones de Foc entre regiones pueden variar, se requiere caracterizar la diversidad genética del hongo entre cultivares y entre regiones del país, con lo cual, se beneficiarán los programas de fitomejoramiento, al tener un conocimiento más amplio de su biología.

La coordinación y la cooperación entre instituciones gubernamentales y de investigación es primordial, para establecer los protocolos necesarios para una oportuna y correcta detección del hongo, incluyendo, a la raza presente.

Conflicto de intereses: El manuscrito fue preparado y revisado con la participación de todos los autores, quienes declaramos que no existe conflicto de intereses que ponga en riesgo la validez de los resultados presentados.

\section{REFERENCIAS}

1. AGUILAR, J. 2013. Improvement of Cavendish banana cultivars through conventional breeding. Acta Horticulturae. 986:205208. https://doi.org/10.17660/ActaHortic.2013.986.21

2. AKILA, R.; RAJENDRAN, L.; HARISH, S.; SAVEETHA, K.; RAGUCHANDER, T.; SAMIYAPPAN, R. 2011. Combined application of botanical formulations and biocontrol agents for the management of Fusarium oxysporum f. sp. cubense (FOC) causing Fusarium wilt in banana. Biological Control. 57(3):175-183. https://doi. org/10.1016/j.biocontrol.2011.02.010

3. AL-HETAR, M.Y.; ZAINAL, M.A.; SARIAH, M.; WONG, M.Y. 2011. Antifungal activity of Chitosan against Fusarium oxysporum f. sp. cubense. J. Applied Polymer Science, 120(4):2434-2439. https://doi.org/10.1002/app.33455

4. ALARCÓN, J.J. 2007. Enfermedades en la producción de Heliconias en los departamentos de Caldas, Risaralda y Quindío. Agronomía. 15(1):45-61.

5. AMIR, H.; ALABOUVETTTE, C. 1993. Involvement of soil abiotic factors in the mechanisms of soil suppressiveness to Fusarium wilts. Soil Biology and Biochemistry (United Kingdom). 25(2):157-164. https://doi.org/10.1016/0038$07179390022-4$

6. ÁNGEL-GARCÍA, C.; ROBLEDO-BURITICÁ, J.; CASTAÑO-ZAPATA, J. 2018. Comparación de métodos de inoculación de Fusarium solani f. sp. passiflorae en plántulas de maracuyá (Passiflora edulis f. flavicarpa). Rev. U.D.C.A. Act. \& Div. Cient. 21(1):23-31. https://doi.org/10.31910/rudca. v21.n1.2018.659

7. BELGROVE, A.; STEINBERG, C.; VILJOEN, A., 2011. Evaluation of nonpathogenic Fusarium oxysporum and Pseudomonas fuorescens for Panama disease control. Plant
Disease (USA). 95(8):951-959. http://doi.org/10.1094/ PDIS-06-10-0409

8. BORGES, A.; VILAR, A.; MATOS, A.; DA SILVA, F. 2007. Redução do mal-do-panamá em bananeira-maçã por inoculação de fungo micorrízico arbuscular. Pesquisa Agropecuária Brasileira. 42(1):35-41. https://dx.doi. org/10.1590/S0100-204X2007000100005

9. BUDDENHAGEN, I. 2009. Understanding strain diversity in Fusarium oxysporum f. sp. cubense and history of introduction of "Tropical Race 4" to better manage banana production. Acta Horticulturae. 828:193-204. https://doi. org/10.17660/ActaHortic.2009.828.19

10. CASTAÑO-ZAPATA, J. 1994. Principios básicos de fitopatología. Editorial Zamorano Academic Press (Honduras). 518p.

11. CASTAÑO-ZAPATA, J. 2002. Principios básicos de fitoepidemiología. Editorial Universidad de Caldas (Colombia). 396p.

12. COSTA, S.N.; BRAGANCA, C.A.D.; RIBEIRO, L.R.; AMORIM, E.P.; OLIVEIRA, S.A.S.; DITA, M.A.; LARANJEIRA, M.M.; HADDAD, F. 2014. Genetic structure of Fusarium oxysporum f. sp. cubense in different regions from Brazil. Plant Pathology, 64(1):1-11. https:// doi.org/10.1111/ppa.12242

13. DALE, J.L.; JAMES, A.; PAUL, J.Y.; KHANNA, H.; SMITH, M.; PERAZA-ECHEVERRIA, S.; GARCÍA-BASTIDAS, F.; KEMA, G.; WATERHOUSE, P.; MENGERSEN, K.; HARDING, R. 2017. Transgenic Cavendish bananas with resistance to Fusarium wilt tropical race 4. Nature Communications (United Kingdom). 8(1):1496. https:// doi.org/10.1038/s41467-017-01670-6

14. DANE, F.; DALGIC, O. 2005. The effects of fungicide benomyl (benlate) on growth and mitosis in onion (Allium cepa L.) root apical meristem. Acta Biológica Hungarica, 56(1):119128. https://doi.org/10.1556/ABiol.56.2005.1-2.12

15. DELTOUR, P.; FRANÇA, S.; PEREIRA, O.; CARDOSO, I.; DE NEVE, S.; DEBODE, J.; HÖFTE, M. 2017. Disease suppressiveness to Fusarium wilt of banana in an agroforestry system: Influence of soil characteristics and plant community. Agriculture, Ecosystems \& Environment. 239:173-181. https://doi.org/10.1016/j.agee.2017.01.018

16. DITA, M.; GARMING, H.; VAN DEN BERGH, I.; STAVER, C.; LESCOT, T. 2013. Banana in Latin American and the Caribbean: Current state challenges and perspectives. Acta Horticulturae. 986(986):365-380. https://doi. org/10.17660/ActaHortic.2013.986.39 
17. FISHAL, E.; MEON, S.; YUN, W. 2010. Induction of tolerance to Fusarium wilt and defense-related mechanisms in the plantlets of susceptible Berangan banana pre-inoculated with Pseudomonas sp. (UPMP3) and Burkholderia sp. (UPMB3). Agricultural Science in China. 9(8):1140-1149. https://doi.org/10.1016/S1671-2927(09)60201-7

18. FORSYTH, L.; SMITH, L.; AITKEN, E. 2006. Identification and characterization of non-pathogenic Fusarium oxysporum capable of increasing and decreasing Fusarium wilt severity. Mycological Research. 110(8):929-935. https:// doi.org/10.1016/j.mycres.2006.03.008

19. FORTUNATO, A.; RODRIGUES, F.; BARONI, J.; SOARES, G.; RODRIGUEZ, M.; PEREIRA, O. 2012. Silicon suppresses Fusarium wilt development in banana plants. J. Phytopathology (United Kingdom). 160(11-12):667-674. https://doi.org/10.1111/jph.12005

20. FRAVEL, D.; OLIVAIN, C.; ALABOUVETTE, C. 2003. Fusarium oxysporum and its biocontrol. New phytologist (United Kingdom). 157:493-502. https://doi.org/10.1046/ j.1469-8137.2003.00700.x

21. GETHA, K.; VIKINESWARY, S. 2002. Antagonistic effects of Streptomyces violaceusniger strain G10 on Fusarium oxysporum $\mathrm{f}$. sp. cubense race 4: indirect evidence for the role of antibiosis in the antagonistic process. J. Industrial Microbiology and Biotechnology (Germany). 28(6):303-310. https://doi. org/10.1038/sj/jim/7000247

22. GHAG, S.B.; SHEKHAWAT, U.K.S.; GANAPATHI, T.R. 2014. Transgenic banana plants expressing a Stellaria media defensin gene (Sm-AMP-D1) demonstrate improved resistance to Fusarium oxysporum. Plant Cell Tiss. and Organ Cult., 119(2):247-255. https://doi.org/10.1007/s11240014-0529-x

23. GNANASEKARAN, P.; SALIQUE, M.; PANNEERSELVAM, A.; UMAMAGHESWARI, K. 2015. In vitro biological control of Fusarium oxysporum $\mathrm{f}$. sp. cubense by using some Indian medicinal plants. Internal J. Current Res. and Academic Review (India). 3(11):107-116.

24. GOPI, M.; THANGAVELU, R. 2014. Suppression of Fusarium wilt disease of banana by Zimmu (Allium cepa L. x Allium sativum L.) leaf extract. African J. Microbiology Res. 8(31):2904-2915. https://doi.org/10.5897/ AJMR2014.6810

25. GORDON, T.R. 2017. Fusarium oxysporum and the Fusarium wilt syndrome. Ann. Rev. Phytopathology. 55:23-39. https:// doi.org/10.1146/annurev-phyto-080615-095919

26. HENNESSY, C.; WALDUCK, G.; DALY, A.; PADOVAN, A., 2005. Weed hosts of Fusarium oxysporum $\mathrm{f}$. sp. cubense tropical race 4 in northern Australia. Australasian Plant Pathology. 34(1):115-117. https://doi.org/10.1071/AP04091

27. HERBERT, J.; MARX, D. 1990. Short-term control of Panama disease of bananas in South Africa. Phytophylactica (South Africa). 22(3):339-340.

28. HERMANTO, C.; DJATNIKA, E.I.; EMILDA, D.; MUJIMAN, S. 2012. Pre-planting treatments for management of banana Fusarium wilt. ARPN J. Agricultural and Biological Science (USA). 7(4):260-265.

29. HUANG, Y.H.; WANG, R.C.; LI, C.H.; ZUO, C.W.; WEI, Y.R.; ZHANG, L.; YI, G.J. 2012. Control of Fusarium wilt in banana with Chinese leek. European Journal of Plant Pathology / European Foundation for Plant Pathology: 134(1):87-95. http://doi.org/10.1007/s10658-012-0024-3

30. JIMÉNEZ, R.; JIMÉNEZ, M. 2011. Integrated management of Fusarium wilt diseases. In: Alves-Santos, F.M; Diez, J.J. (Eds). Control of Fusarium Diseases, Chapter: Chapter 7. Integrated Management of Fusarium Wilt Diseases. Research Signpost. p.177-215. Disponible desde Internet en: https://www.researchgate.net/publication/249009577_ Integrated_Management_of_Fusarium_Wilt_Diseases (con acceso 30/06/2018).

31. JUNIAWAN; ROCHDJATUN, I.; DJAUHARI, S.; HIMAWAN, T. 2016. Inhibition test of clove leaf oils to Fusarium oxysporum f. sp. cubense. IOSR J. Agriculture and Veterinary Science. 9(4):1-7. http://doi.org/10.9790/23800904010107

32. KIDANE, E.G.; LAING, M.D. 2010. Integrated control of Fusarium wilt of banana (Musa spp.). Acta Horticulturae (Belgium). 879:315-321. https://doi.org/10.17660/ ActaHortic.2010.879.32

33. KÖBERL, M.; DITA, M.; MARTINUZ, A.; STAVER, C.; BERG, G. 2017. Members of Gammaproteobacteria as indicator species of healthy banana plants on Fusarium wilt-infested fields in Central America. Scientific Reports. 7: 45318. https://doi.org/10.1038/srep45318

34. LAKSHMANAN, P.; SELVARAJ, P.; MOHAN, S. 1987. Efficacy of different methods for the control of Panama disease. Tropical Pest Management (United Kingdom). 33(4):373374. http://dx.doi.org/10.1080/09670878709371187

35. LESLIE, J.; SUMMERELL, B. 2006. The Fusarium laboratory manual. Blackwell Publishing. Iowa, USA. 387p.

36. LI, X.; BAI, T.; LI, Y.; RUAN, X.; LI, H. 2013. Proteomic analysis of Fusarium oxysporum f. sp. cubense tropical race 4 - inoculated response to Fusarium wilts in the 
banana root cells. Proteome Science. 11:41. https://doi. org/10.1186/1477-5956-11-41

37. LI, W.; DITA, M.; WU, W.; HU, G.; XIE, J.; GE, X. 2014. Resistance sources to Fusarium oxysporum f. sp. cubense tropical race 4 in banana wild relatives. Plant Pathology. 64(5):1061-1067. https://doi.org/10.1111/ppa.12340

38. LIAN, J.; WANG, Z.; ZHOU, S. 2008. Response of endophytic bacterial communities in banana tissue culture plantlets to Fusarium wilt pathogen infection. J. General and Applied Microbiology (Japan). 54(2):83-92. https://doi. org $/ 10.2323 /$ jgam.54.83

39. LÓPEZ, N.; CASTAÑO-ZAPATA, J. 2012. Characterization of phytopathogenic fungi, bacteria, nematodes and viruses in four commercial varieties of Heliconia (Heliconia sp.). Rev. Facultad Nacional de Agronomía Medellín. 65(2):66976710.

40. MARÍN-SERNA, S.M.; GONZÁLEZ-GUZMÁN, J.J.; CASTAÑO-ZAPATA, J.; CEBALLOS-AGUIRRE, N. 2014. Respuesta de quince introducciones de tomate tipo cereza (Solanum spp.) a la marchitez vascular (Fusarium oxysporum f. sp. 1ycopersici Snyder \& Hansen). Agronomía. 22(2):48-50.

41. MELDRUM, R.A.; DALY, A.M.; TRAN-NGUYEN, L.T.T.; AITKEN, E.A.B. 2013a. ¿Are banana weevil borers a vector in spreading Fusarium oxysporum f. sp. cubense tropical race 4 in banana plantations? Australasian Plant Pathology. 42:543-549. https://doi.org/10.1007/s13313-013-0214-2

42. MELDRUM, R.A.; DALY, A.M.; TRAN-NGUYEN, L.T.T.; AITKEN, E.A.B. 2013b. The effect of surface sterilants on spore germination of Fusarium oxysporum $\mathrm{f}$. $\mathrm{sp}$. cubense tropical race 4. Crop Protection. 54:194-198. https://doi. org/10.1016/j.cropro.2013.08.014

43. MENGANE, S.; KAMBLE, S. 2014. Bioefficacy of plant extracts on Fusarium oxysporum f. sp. cubense causing panama wilt of banana. IJPBS (India). 4(3):24-27.

44. MOHANDAS, S.; MANJULA, R.; RAWAL, R.D.; LAKSHMIKANTHA, H.C.; CHAKRABORTY, S.; RAMACHANDRA, Y.L. 2010. Evaluation of arbuscular mycorrhiza and other biocontrol agents in managing Fusarium oxysporum f. sp. cubense infection in banana cv. Neypoovan. Biocontrol Science and Technology (United Kingdom). 20(2):165-181. https://doi. org/10.1080/09583150903438439

45. MOLINA, A.B.; FABREGAR, E.G.; RAMILLETE, E.G.; SINOHIN, V.O.; VILJOEN, A. 2011. Field resistance of selected banana cultivars against tropical race 4 of Fusarium oxysporum f. sp. cubense in the Philippines. Phytopathology
(United States). 101:S122. https://doi.org/10.13140/ RG.2.2.16107.23844

46. MOLINA, A.; SINOHIN, V.; FABREGAR, E.; RAMILLETE, E.B.; LOAYAN, M.; CHAO, C. 2016. Field resistance of Cavendish somaclonal variants and local banana cultivars to tropical race 4 of Fusarium wilt in the Philippines. Acta Horticulturae 1114:227-230. https://doi.org/10.17660/ ActaHortic.2016.1114.31

47. MONTEIRO, F.; FERREIRA, L.; SILVA, J.; PACHECO, L.; SOUZA, P. 2013. Influence of plant extracts and essential oils against Panama disease (Fusarium oxysporum f. sp. cubense) in banana seedlings. J. Agricultural Science (Canadá). 5(4):63-74. http://dx.doi.org/10.5539/jas.v5n4p63

48. NEL, B.; STEINBERG, C.; LABUSCHAGNE, N.; VILJOEN, A. 2007. Evaluation of fungicides and sterilants for potential application in the management of Fusarium wilt of banana. Crop Protection (United Kingdom). 26(4):697705. https://doi.org/10.1016/j.cropro.2006.06.008

49. PATTISON, A.B.; WRIGHT, C.L.; KUKULIES, T.L.; MOLINA, A.B. 2014. Ground cover management alters development alters development of Fusarium wilt symptoms in Ducasse bananas. Australasian Plant Pathology. 43(4):465-476. https://doi.org/10.1007/s13313-014-0296-5

50. PEI, X.W.; CHEN, S.K.; WEN, R.M.; YE, S.; HUANG, J.Q.; ZHANG, Y.Q.; WANG, B.S.; WANG, Z.X.; JIA, S.R. 2005. Creation of transgenic bananas expressing human lysozyme gene for Panama wilt resistance. J. Int. Plant Biol. 47(8):971997. https://doi.org/10.1111/j.1744-7909.2005.00141.x

51. PENG, H.X.; SIVASITHAMPARAM, K.; TURNER, D.W. 1999. Chlamydospore germination and Fusarium wilt of banana plantlets in suppressive and conducive soils are affected by physical and chemical factors. Soil Biology and Biochemistry (United Kingdom). 31(10):1363-1374. https://doi.org/10.1016/S0038-0717(99)00045-0

52. PÉREZ, L.; DITA, M.; MARTINEZ, E. 2014. Prevention and diagnostic of Fusarium wilt (Panama disease) of banana caused by Fusarium oxysporum f. sp. cubense tropical race 4 (TR4). Proc. Regional Workshop on the Diagnosis of Fusarium Wilt (Panama disease) caused by Fusarium oxysporum f. sp. cubense Tropical Race 4: Mitigating the Threat and Preventing its Spread in the Caribbean, St. Augustine, Trinidad and Tobago. FAO, Rome, Italy. 74p. Disponible desde Internet en: http://www.fao. org/fileadmin/templates/agphome/documents/Pests_ Pesticides/caribbeantr4/13ManualFusarium.pdf (acceso 30/06/2018).

53. PÉREZ, L. 2015. Las mejores prácticas para la prevención de la raza 4 tropical de la marchitez por Fusarium y otras 
enfermedades exóticas en fincas bananeras. Fitosanidad. 19(3):243-250.

54. PERRIER, X.; DE LANGHE, E.; DONOHUE, M.; LENTFER, C.; VRYDAGHS, L.; BAKRY, F.; CARREEL, F.; HIPPOLYTE, I.; HORRY, J.P.; JENNY, C.; LEBOT, V.; RISTERUCCI, A.M.; TOMEKPE, K.; DOUTRELEPONT, H.; BALL, T.; MANWARING, J.; DE MARET, P.; DENHAM, T. 2011. Multidisciplinary perspectives on banana (Musa spp.) domestication. Proc. Nal Acad. Sciences (USA). 108(28):11311-11318. https:// doi.org/10.1073/pnas.1102001108

55. PLOETZ, R.C. 2006. Fusarium wilt of banana is caused by several pathogens referred to as Fusarium oxysporum f. sp. cubense. Phytopathology (USA). 96(6):653-656. https://doi. org/10.1094/PHYTO-96-0653

56. PLOETZ, R.C. 2015a. Management of Fusarium wilt of banana: A review with special reference to tropical race 4 . Crop Protection (United Kingdom). 73:7-15. http://dx.doi. org/10.1016/j.cropro.2015.01.007

57. PLOETZ, R.C. 2015b. Fusarium wilt of banana. Phytopathology (USA) 105(12):1512-1521. https://doi.org/10.1094/ PHYTO-04-15-0101-RVW

58. RAMU, V.; VENKATARANGAIAH, K.; KRISHNAPPA, P.; SHIMOGA RAJANNA, S.K.; DEEPLANAIK, N.; CHANDRA PAL, A.; KINI, K.R. 2016. Identification of biomarkers for resistance to Fusarium oxysporum f. sp. cubense infection and in Silico studies in Musa paradisiaca cultivar Puttabale through proteomic approach. Proteomes. 4(1):9. https://doi.org/10.3390/proteomes4010009

59. SARAVANAN, T.; MUTHUSAMY, M.; MARIMUTHU, T. 2003. Development of integrated approach to manage the Fusarium wilt of banana. Crop Protection (United Kingdom). 228(9):1117-1123. https://doi.org/10.1016/ S0261-2194(03)00146-7

60. SHEN, Z.; ZHONG, S.; WANG, Y.; WANG, B.; MEI, X.; LI, R.; RUAN, Y.; SHEN, Q. 2013. Induced soil microbial suppression of banana Fusarium wilt disease using compost and biofertilizers to improve yield and quality. European J. Soil Biology. 57:1-8. https://doi.org/10.1016/j. ejsobi.2013.03.006

61. SHIRANI, S.; SIJUN, Z. 2018. Banana Fusarium wilt (Fusarium oxysporum $\mathrm{f}$. sp. cubense) control and resistance, in the context of developing wilt- resistant bananas within sustainable production systems. Horticultural Plant Journal. 4(5):208218. https://doi.org/10.1016/j.hpj.2018.08.001

62. SIDDHESH, B.; UPENDRA, K.; THUMBALLI, R. 2015. Fusarium wilt of banana: biology, epidemiology and management. Internal J. Pest Management. 61(3):250-263. https://doi.org/10.1080/09670874.2015.1043972

63. SMITH, M.; LANGDON, P.; PEGG, K.; DANIELLS, J. 2014. Growth, yield and Fusarium wilt resistance of six FHIA tetraploid bananas (Musa spp.) grown in the Australian subtropics. Scientia Horticulturae. 170:176-181. https:// doi.org/10.1016/j.scienta.2014.02.029

64. SUBRAMANIAM, S.; MAZIAH, M.; SARIAH, M.; PUAD, M.P.; XAVIER, R. 2006. Bioassay method for testing Fusarium wilt disease tolerance in transgenic banana. Scientia Horticulturae. 108(4):378-389. https://doi. org/10.1016/j.scienta.2006.02.028

65. SUBRAMANYAM, K.; SUBRAMANYAM, K.; SAILAJA, K.V.; SRINIVASULU, M.; LAKSHMIDEVI, K. 2011. Highly efficient Agrobacterium mediated transformation of banana cv. Rasthali (AAB) via sonication and vacuum infiltration. Plant Cell Rep. 30(3):425-436. http://dx.doi. org/10.1007/s00299-010-0996-4

66. SUN, B.; PENG, M.; WANG, Y.G.; ZHAO, P.J.; XIA, Q.Y. 2011. Isolation and characterization of antagonistic bacteria against Fusarium wilt and induction of defense related enzymes in banana. African J. Microbiology Res. 5(5):509515. https://doi.org/10.5897/AJMR10.607

67. THANGAVELU, R.; JAYANTHI, A. 2009. A RFLP analysis of rDNA-ITS regions of native non-pathogenic Fusarium oxysporum isolates and their field evaluation for the suppression of Fusarium wilt disease of banana. Australasian Plant Pathology. 38(13):13-21. https://doi. org/10.1071/AP08071

68. THANGAVELU, R.; GOPI, M. 2015. Field suppression of Fusarium wilt disease in banana by the combined application of native endophytic and rhizospheric bacterial isolates possessing multiple functions. Phytopathologia Mediterranea (Italy) 54(2):241-252. https://doi. org/10.14601/Phytopathol_Mediterr-15160

69. TING, A.S.Y.; MEON, S.; KADIR, J.; RADU, S.; SINGH, G. 2010. Induction of host defense enzymes by the endophytic bacterium Serratia marcescens, in banana plantlets. Internal J. Pest Management (United Kingdom). 56(2):183-188. https://doi.org/10.1080/09670870903324198

70. TING, A.S.Y.; MAH, S.W.; TEE, C.S. 2010. Identification of volatile metabolites from fungal endophytes with biocontrol potential towards Fusarium oxysporum F. sp. cubense Race 4. American J. Agricultural and Biological Sciences. 5(2): 177182. https://doi.org/10.3844/ajabssp.2010.177.182

71. VAN DER PLANK, J.E. 1963. Plant diseases: epidemics and control. Academic Press. New York. 349p. 
72. VILLA, A.; PÉREZ, R.; MORALES, H.; BASURTO, M.; SOTO, J.; MARTÍNEZ, E. 2015. Situación actual en el control de Fusarium spp. y evaluación de la actividad antifúngica de extractos vegetales. Acta Agronómica. 64(2):194-205. https://dx.doi.org/10.15446/acag.v64n2.43358

73. WANG, B.; YUAN, J.; ZHANG, F.; SHEN, Z.; ZHANG, F; LI, R.; RUAN, Y.; SHEN, Q. 2013. Effects of novel bioorganic fertilizer produced by Bacillus amyloliquefaciens W19 on antagonism of Fusarium wilt of banana. Biology and Fertility Soils (Germany). 49(4):435-446. https://doi. org/10.1007/s00374-012-0739-5

74. WANG, Z.; JIA, C.; LI, J.; HUANG, S.; XU, B.; JIN, Z. 2014. Activation of salicylic acid metabolism and signal transduction can enhance resistance to Fusarium wilt in banana (Musa acuminate L. AAA group, cv. Cavendish). Funct. Integr. Genomics. 15:47-62. https://doi. org/10.1007/s10142-014-0402-3

75. WANG, B.; LI, R.; RUAN, Y.; OU, Y.; ZHAO, Y.; QIRONG, S. 2015. Pineapple-banana rotation reduced the amount of Fusarium oxysporum more than maize-banana rotation mainly through modulating fungal communities. Soil Biology \& Biochemistry. 86:77-86. http://dx.doi.org/10.1016/j. soilbio.2015.02.021

76. WANG, B.; SHEN, Z.; ZHANG, F.; RAZA, W.; YUAN, J.; HUANG, R.; RUAN, Y.; LI, R.; SHEN, Q. 2016. Bacillus amyloliquefaciens strain W19 can promote growth and yield and suppress Fusarium wilt in banana under greenhouse and field conditions. Pedosphere. 26(5):733-744. https:// doi.org/10.1016/S1002-0160(15)60083-2

77. WEBER, O.B.; MUNIZ, C.R.; VITOR, A.O.; FREIRE, F.C.; OLIVEIRA, V.M. 2007. Interaction of endophytic diazotrophic bacteria and Fusarium oxysporum f. sp. cubense on plantlets of banana 'Maça.'. Plant and Soil (Netherlands). 298(1):47-56. https://doi.org/10.1007/s11104-007-9335-0

78. WEN, T.; HUANG, X.; ZHANG, J.; ZHU, T.; MENG, L.; CAI, Z. 2015. Effects of water regime, crop residues, and application rates on control of Fusarium oxysporum f. sp. cubense. J. Environmental Sciences. 31:30-37. https://doi. org/10.1016/j.jes.2014.11.007

79. WU, Y.; YI, G.; PENG, X.; HUANG, B.; LIU, E.; ZHANG, J. 2013. Systemic acquired resistance in Cavendish banana induced by infection with an incompatible strain of Fusarium oxysporum f. sp. cubense. J. Plant Physiol. 170:10391046. https://doi.org/10.1016/j.jplph.2013.02.011
80. ZACKY, F.A.; TING, A.S.Y. 2013. Investigating the bioactivity of cells and cell-free extracts of Streptomyces griseus towards Fusarium oxysporum f. sp. cubense race 4. Biological Control (United States). 66(3):204-208. https://doi.org/10.1016/j. biocontrol.2013.06.001

81. ZHANG, M.; ZHANG, M.; CHEN, P.; RUAN, Y.; ZHU, Y.; SHEN, Q. 2013a. Influence of nitrate/ammonium ratio on the plant growth of banana and related wilt disease development. J. Plant Nutrition and Fertilizer (China). 19(5):1241-1247. https://doi.org/10.11674/ zwyf.2013.0525

82. ZHANG, H.; MALLIK, A.; ZENG, R.S.J. 2013b. Control of Panama disease of banana by rotating and intercropping with Chinese chive (Allium tuberosum Rottler): Role of plant volatiles. Chemical Ecology. 39(2):243-252. https://doi. org/10.1007/s10886-013-0243-x

83. ZHANG, N.; WU, K.; HE, X.; LI, S.; ZHANG, Z.; SHEN, B.; YANG, X.; ZHANG, R.; HUANG, Q.; SHEN, Q. 2014a. A new bioorganic fertilizer can effectively control banana wilt by strong colonization with Bacillus subtilis N11. Plant and Soil. 344(1-2):87-97. https://doi.org/10.1007/s11104011-0729-7

84. ZHANG, N.; HE, X.; ZHANG, J.; RAZA, W.; YANG, X.; RUAN, Y.; SHEN, Q.; HUANG, Q. 2014b. Suppression of Fusarium wilt of banana with application of bioorganic fertilizers. Pedosphere. 24(5):613-624. https://doi. org/10.1016/S1002-0160(14)60047-3

85. ZUO, C.; LI, C.; LI, B.; WEI, Y.; HU, C.; YANG, Q.; YANG, J.; SHENG, O.; KUANG, R.; DENG, G.; KUMAR, R.; YI, G. 2015. The toxic mechanism and bioactive components of Chinese leek root exudates acting against Fusarium oxysporum f. sp. cubense tropical race 4. European J. Plant Pathology. 143(3):447-460. https://doi.org/10.1007/ s10658-015-0697-5

86. ZUO, C.; DENG, G.; LI, B.; HUO, H.; LI, CH.; HU, CH.; KUANG, R.; YANG, Q.; DONG, T.; SHENG, O.; YI, G. 2018. Germplasm screening of Musa spp. for resistance to Fusarium oxysporum f. sp. cubense tropical race 4 (FOC TR4). European J. Plant Pathology. 151(3):723-734. https://doi. org/10.1007/s10658-017-1406-3 